\title{
Laboratory diagnosis of paediatric tuberculosis in the European Union/European Economic Area: analysis of routine laboratory data, 2007 to 2011
}

\author{
A Sanchini (sanchinia@rki.de) ${ }^{1,2}$, L Fiebig² ${ }^{2}$ F Drobniewski ${ }^{3,4}$, W Haas $^{1}$, E Richter ${ }^{5}$, V Katalinic-Jankovic ${ }^{6}$, E Pimkina ${ }^{7}$, G Skenders ${ }^{8}$, \\ D M Cirillo9, European Reference Laboratory Network for TB (ERLN-TB) members ${ }^{10}$, Y Balabanova $^{1,3}$ \\ 1. Department for Infectious Disease Epidemiology, Robert Koch Institute, Berlin, Germany \\ 2. European Public Health Microbiology Training Programme (EUPHEM), European Centre for Disease Prevention and Control \\ (ECDC), Stockholm, Sweden \\ 3. Queen Mary College, Barts and the London School of Medicine, University of London, London, United Kingdom \\ 4. National Mycobacterium Reference Laboratory, Public Health England, London, United Kingdom \\ 5. National Reference Center for Mycobacteria, Forschungszentrum Borstel, Borstel, Germany \\ 6. National Mycobacterium Reference Laboratory, Croatian National Institute of Public Health, Zagreb, Croatia \\ 7. Infectious Diseases and Tuberculosis Hospital, Affiliate of Vilnius University Hospital Santariskiu Klinikos, Vilnius, \\ Lithuania \\ 8. Riga East University Hospital, Centre of Tuberculosis and Lung Diseases, Riga, Latvia \\ 9. San Raffaele Scientific Institute, Milan, Italy \\ 10. Members of the network are listed at the end of the article
}

Sanchini A, Fiebig L, Drobniewski F, Haas W, Richter E, Katalinic-Jankovic V, Pimkina E, Skenders G, Cirillo DM, European Reference Laboratory Network for TB (ERLN-TB) members, Balabanova Y. Laboratory diagnosis of paediatric tuberculosis in the European Union/European Economic Area: analysis of routine laboratory data, 2007 to 2011. Euro Surveill. 2014;19(11):pii=20744. Available online: http://www.eurosurveillance.org/ViewArticle.aspx?Articleld=20744

Laboratory confirmation of paediatric tuberculosis (TB) is frequently lacking. We reviewed the range of routine laboratory tests and their performance in different biological samples used to diagnose active TB in children. A questionnaire-based survey was conducted among the European Reference Laboratory Network for TB followed by collection of routine laboratory data on 10,549 paediatric samples tested in 2007 to 2011 at six reference laboratories (in Croatia, Germany, Italy, Latvia, Lithuania and the United Kingdom (UK)). The questionnaire showed that all laboratories used rapid assays. Non-respiratory samples were collected more often in Germany (135/275, $49.1 \%)$ and the UK $(490 / 2,140,22.9 \%)$ compared with Croatia (138/2,792, 4.9\%), Latvia (222/2,401, 9.2\%) and Lithuania $(76 / 1,549,4.9 \%)$. Overall laboratory positivity rates (isolation of Mycobacterium tuberculosis complex and/or identification of its nucleic acids in a sample) were higher in lymph node and gastric aspirate samples (14/203 (6.9\%) and 43/1,231 (3.5\%)) than in sputum samples $(89 / 4,684(1.9 \%))$. Pooled sensitivity, specificity, positive and negative predictive values and accuracy of molecular assays assessed against solid or liquid culture were $79.2 \%, 93.6 \%, 67.1 \%$, $96.5 \%$ and $91.6 \%$, respectively. A more intensive approach in obtaining gastric aspirate and non-respiratory samples may increase laboratory confirmation of paediatric TB. Major effort is needed in optimisation and validation of molecular tests in these samples.

\section{Introduction}

Tuberculosis (TB) affects globally about 490,000 children under 15 years-old, with 64,000 related deaths occurring every year [1]. Paediatric TB is an indicator of recent transmission in the population. Often, children experience more severe forms of the disease, such as miliary or meningeal TB $[2,3]$. Young children are rarely able to expectorate sputum; therefore, other respiratory samples, such as gastric aspirates (GA) or bronchoalveolar lavages (BAL), can be obtained for diagnostic purposes, although these procedures are more unpleasant for a child than sputum collection $[1,3-5]$. Collection of non-respiratory samples (lymph node (LN), pus or tissue biopsy) is necessary to diagnose extrapulmonary TB; however, these procedures are relatively invasive $[6,7]$.

TB in children often has a paucibacillar nature, resulting in microscopy smears that are negative $[3,4,8]$. Culture isolation, a more sensitive method, takes up to 14 days due to slow growth of Mycobacterium tuberculosis complex (MTB(C)) bacteria; however, even culture is seldom positive in paediatric specimens due to very few bacilli present in a sample $[3,4,8]$. Further full drug susceptibility testing (DST) can only be done once an isolate is available and takes two more weeks $[9,10]$.

Consequently, the diagnosis of paediatric TB often relies on a combination of clinical judgment and radiological findings, prompting initiation of treatment without clear laboratory evidence $[1,8,9,11,12]$. Unlike laboratory criteria, there is as yet no universally applied diagnostic algorithm based on clinical and radiological criteria that are objective $[8,11,12]$. 
The most important advance in TB diagnosis is the introduction of the internationally endorsed molecular assay Xpert MTB/RIF (Cepheid Inc., Sunnyvale, CA, United States), which identifies MTB and rifampicin resistance from sputum samples [13]. A growing body of evidence supports the adequacy of Xpert MTB/RIF for adult TB diagnosis [14-16].

Recent studies have investigated the performance of the Xpert MTB/RIF system in children and showed promising results when the test is applied to sputum, nasopharyngeal aspirates (NPA), GA or non-respiratory samples $[4,6,7,17-19]$. However, there are limited data on the usefulness of molecular assays for paediatric TB diagnosis in Europe, where TB prevalence is low [20].

The aim of the study was to give an overview of the range of routine diagnostic tests and their performance for different types of samples used to diagnose active TB in children across European laboratories.

\section{Methods}

\section{Study design}

The study was planned in two stages. Firstly, we invited all (38) national TB reference laboratories across the European Union (EU)/European Economic Area (EEA) countries that are members of the European Reference Laboratory Network for TB (ERLN-TB) - an initiative supported by the European Centre for Disease Prevention and Control (ECDC) [21] - to participate in a questionnaire-based survey. The questionnaire aimed to determine the variety of algorithms used to diagnose paediatric TB and the type of samples collected from children; it contained 53 questions and was based on an earlier questionnaire used across the ERLN-TB [22].

Secondly, six laboratories of the network (referred to hereafter as study sites) agreed to provide their routine data for all consecutive primary samples and reference cultures referred for diagnosis from children younger than 15 years-old with suspected TB during 2007 to 2011.

Primary samples were defined as specimens referred to a reference laboratory for primary diagnostics, while reference cultures were cultures isolated by local laboratories and referred to a reference laboratory for confirmation, DST or molecular typing.

Low-incidence western and central European settings were represented by the following laboratories: the National Reference Centre for Mycobacteria at Forschungszentrum Borstel, Borstel, Germany; San Raffaele Scientific Institute, in collaboration with the Institute 'Villa Marelli', Niguarda Ca' Granda Hospital, Milan, Italy; the National Mycobacterium Reference Laboratory, Public Health England, London, United Kingdom (UK) and the National Mycobacterium Reference Laboratory, National Institute of Public Health, Zagreb, Croatia (TB incidence in 2012 being
$5.6,6.7,15.0$ and 14.0 cases per 100,000 population in the respective countries [23]).

The National TB Reference Laboratory at the Latvian Infectology Centre, Upeslejas, Latvia, and the Tuberculosis Bacteriology Laboratory at the Infectious Diseases and Tuberculosis Hospital, Vilnius, Lithuania, represented medium-incidence eastern European countries, with TB incidence in 2012 being 53.0 and 66.0 cases per 100,000 population, respectively [23].

Only partial data were available from the Italian site for 2011; this is the only site that does not have a national reference laboratory function, covering mainly the Lombardy region of Italy. Germany provided data for 2011 only. We did not exclude control samples, as there were only a few of those.

The laboratory data were collected using an Excelbased tool, with line-listing by sample. The Croatian, German, Italian and UK sites received a small proportion of all paediatric samples in the country (estimated by the laboratory directors as less than $10 \%$ ), while the Lithuanian site covered approximately half and the Latvian site all paediatric samples in their countries.

Laboratory positivity was confirmed by a positive culture (on solid or liquid media) identified as $\mathrm{MTB}(\mathrm{C})$ or non-tuberculous mycobacteria (NTM) and/or by the identification of MTB(C) or NTM nucleic acid directly in a sample by a molecular assay.

\section{Ethics statement}

Routine laboratory data were sent to the Robert Koch Institute, Berlin, Germany, without personal identifiers. The study obtained a waiver of informed consent and ethics review permission from the Robert Koch Institute.

\section{Data analysis}

Per-sample and per-patient analyses were conducted. Samples were categorised as 'respiratory' (sputum, $B A L, G A$, pleural fluid and other respiratory samples such as NPA), 'non-respiratory' (cerebrospinal fluid (CSF), LN, pus, blood, other tissue biopsy, urine and other non-respiratory samples such as pericardial fluid) and 'unknown' (where exact information on sample type was missing from the records).

Calculations related to the $\operatorname{MTB}(\mathrm{C})$ did not include the Mycobacterium bovis strain used in the Bacillus Calmette-Guérin (BCG) vaccine.

The incremental positivity rate, defined as additional sensitivity gained by testing more than one sample from the same patient, was calculated for GA samples.

Associations between correct diagnostic yield and sample type were tested using Pearson's chi-squared test or Fisher's exact test. Statistical tests were twosided at alpha $=0.05$. 
Laboratory diagnosis of paediatric tuberculosis: types of paediatric primary samples received across the six study sites, 2007-2011 (n=9,157)

\begin{tabular}{|c|c|c|c|c|}
\hline \multirow[b]{2}{*}{ Site } & \multirow{2}{*}{$\begin{array}{c}\text { Total number of samples } \\
\text { received }\end{array}$} & \multicolumn{3}{|c|}{ Sample type } \\
\hline & & $\begin{array}{l}\text { Respiratory }{ }^{\mathrm{a}} \\
\mathrm{n}(\%)\end{array}$ & $\begin{array}{c}\text { Non-respiratory } \\
n(\%)\end{array}$ & $\begin{array}{l}\text { Unknown } \\
\text { n (\%) }\end{array}$ \\
\hline $\begin{array}{l}\text { Croatia } \\
\text { CIPH }\end{array}$ & 2,792 & $2,650(94.9)$ & $138(4 \cdot 9)$ & $4(0.1)$ \\
\hline $\begin{array}{l}\text { Germany } \\
\text { NRCM }\end{array}$ & 275 & 129 (46.9) & $135(49.1)$ & $11(4 \cdot 0)$ \\
\hline $\begin{array}{l}\text { Italy }^{b} \\
\text { HSR }\end{array}$ & 340 & NA & NA & NA \\
\hline $\begin{array}{l}\text { Latvia } \\
\text { NTRL }\end{array}$ & 2,401 & $2,167(90.3)$ & $222(9.2)$ & $12(0.5)$ \\
\hline $\begin{array}{l}\text { Lithuania } \\
\text { TBL }\end{array}$ & 1,549 & $1,464(94 \cdot 5)$ & $76(4.9)$ & $9(0.6)$ \\
\hline $\begin{array}{l}\text { United Kingdom } \\
\text { NMRL }\end{array}$ & 2,140 & $1,643(76.8)$ & $490(22.9)$ & $7(0.3)$ \\
\hline All sites & $9,157^{c}$ & $8,053(87.9)$ & $1,061(11.6)$ & $43(0.5)$ \\
\hline
\end{tabular}

CIPH: Croatian National Institute of Public Health, National Mycobacterium Reference Laboratory; HSR: San Raffaele Scientific Institute in collaboration with the Institute 'Villa Marelli', Niguarda Ca' Granda Hospital; NA: data not available; NMRL: National Mycobacterium Reference Laboratory; NRCM: National Reference Centre for Mycobacteria at Forschungszentrum Borstel; NTRL: National TB Reference Laboratory at the Latvian Infectology Centre; TBL: Tuberculosis Bacteriology Laboratory at the Infectious Diseases and Tuberculosis Hospital.

a Respiratory samples: sputum, gastric aspirate, bronchoalveloar lavage, pleural fluid, other respiratory samples (e.g. nasopharyngeal aspirate). Non-respiratory samples: cerebrospinal fluid, lymph node, pus, other biopsy tissue, urine, other non-respiratory samples (e.g. pericardial fluid)

b Data from 2011 only.

Does not include the 340 samples from Italy.

The results of the different types of molecular tests were pooled since the number of samples did not allow stratified analysis of each test. Sensitivity, specificity, positive and negative predictive values (PPV and NPV, respectively) and the accuracy of the pooled molecular assays was assessed against culture and DST results in samples tested by both methods.

The answers from the survey questionnaire were entered into EpiData (EpiData Association, Odense, Denmark) and analysed using STATA (StataCorp. 2011. Stata Statistical Software: Release 12. College Station, TX: StataCorp LP). The routine laboratory data were collected from the study sites using a Microsoft Excel spreadsheet and analysed in STATA.

\section{Results}

\section{Questionnaire-based survey}

A total of 21 TB reference laboratories from Belgium, Bulgaria, Croatia, Cyprus, Czech Republic, Estonia, Finland, Germany, Greece, Hungary, Italy (two laboratories), Latvia, Lithuania, Norway, Portugal, Slovenia, Spain (two laboratories), Sweden and the UK sent their responses.

The population served by these laboratories varied between 400,000 and 20,000,000 inhabitants. Annually, the laboratories receive a median of 70 (range: 6-950) paediatric primary samples and reference cultures representing a median of 3.1\% (range: $0.3-8.3 \%$ ) of the total laboratory workload.

Of the 21 laboratories, 16 receive primary samples; the Latvian and Lithuanian laboratories receive primary samples only. Five laboratories receive reference cultures only (Belgium, Czech Republic, Finland, Norway and Spain). The most common sample type received from children is sputum (16 laboratories), followed by GA and BAL (15 laboratories each). A total of 11 laboratories reported receiving more than one type of sample from each child.

All laboratories receiving primary samples perform smear microscopy, molecular identification, culture (solid or liquid media, commercial or in-house) and firstand second-line DST. All undergo international quality assurance, demonstrating satisfactory performance.

The most frequently used direct rapid molecular identification assay included Xpert MTB/RIF $(n=10)$ and GenoType MTBDRplus (Hain Lifescience, $\mathrm{GmbH}$, Germany) ( $n=8) ; 10$ laboratories used more than one assay. All laboratories rapidly identified MTB(C) from positive culture using GenoType CM/AS (Hain Lifescience, GmbH, Germany) ( $n=11$ ), BD MGIT TBC Id (Becton Dickinson, United States) and GenoType MTBDRplus assays ( $n=8$ each). Nine laboratories 
Laboratory diagnosis of paediatric tuberculosis: type and percentage of paediatric primary samples received across five study sites, 2007-2011 ( $\mathrm{n}=9,157)$

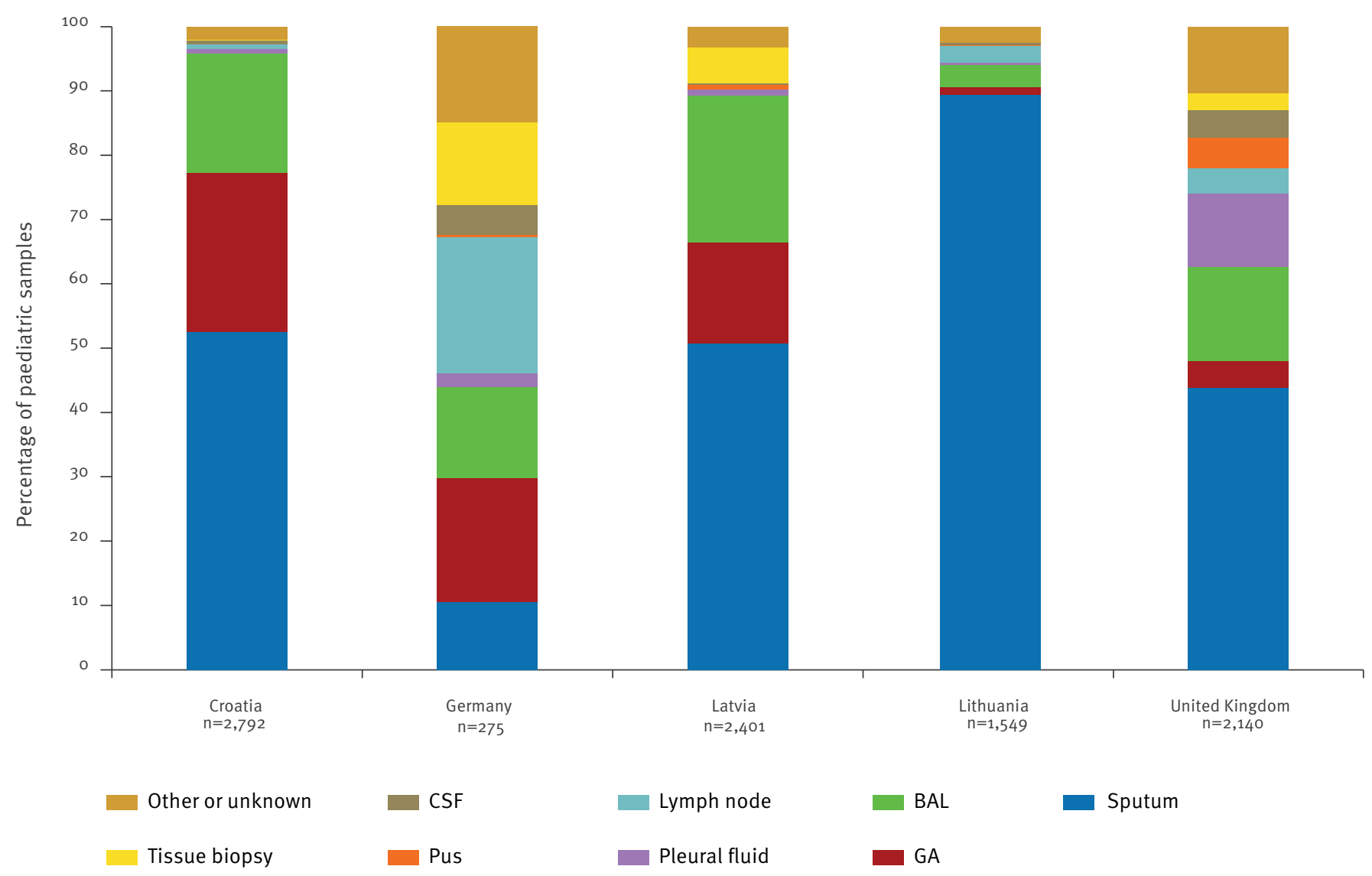

CSF: cerebrospinal fluid; BAL: bronchoalveolar lavage; GA: gastric aspirate.

Data from the Italian laboratory were not included, since available data specified only that the samples were either respiratory or nonrespiratory, without further information on specific sample type.

routinely used GenoType MTBDRsl (Hain Lifescience, Germany) for rapid second-line DST.

In 17 laboratories, the algorithms used to diagnose paediatric and adult TB did not differ; four laboratories reported the use of extra tests for children. In Latvia and Lithuania, for example, when there was a positive culture from a biopsy sample from a child GenoType MTBC (Hain Lifescience, Germany) was used to distinguish between MTB and M. bovis BCG.

\section{Routine laboratory data}

Number and range of primary samples received A total of 9,157 primary samples and 1,392 reference cultures were received from children across all six study sites within the five-year study period (Table 1).

Of all primary samples, the vast majority $(8,053 / 9,157$, $87.9 \%)$ were respiratory, including 4,974 sputum (61.8\%), 1,467 BAL (18.2\%), 1,232 GA (15.3\%) and 298 pleural fluid (3.7\%) samples; 1,061 samples were non-respiratory, including 230 tissue biopsy samples other than LN (21.7\%), 205 LN (19.3\%), 182 urine (17.2\%), 140 CSF (13.2\%) and 118 pus (11.1\%) (Figure).

In the Croatian, Latvian and Lithuanian sites, less than $10 \%$ of all paediatric samples were non-respiratory $(138 / 2,792,4.9 \% ; 222 / 2,401,9.2 \% ; 76 / 1,549,4.9 \%$, respectively), whereas in the UK site, this proportion was higher, 490/2,140 (22.9\%). The German site received more non-respiratory than respiratory primary samples $(135 / 275,49.1 \%$ and $129 / 275,46.9 \%$, respectively), with $\mathrm{LN}$ being the most common sample type $(58 / 275,21.1 \%)$ (Table 1 , Figure).

Microscopy, culture and drug susceptibility testing Most of the primary samples $(8,176 / 9,157,89.3 \%)$ were subjected to smear microscopy, resulting in a $3.3 \%$ $(268 / 8,176)$ positivity rate (Table 2$)$. More non-respiratory than respiratory samples were positive by microscopy $(82 / 816,10.0 \%$ vs $181 / 7,320,2.5 \%$, p<0.0001). 
Laboratory diagnosis of paediatric tuberculosis: tests performed on paediatric primary samples across the six study sites, 2007-2011 (n=9,157)

\begin{tabular}{|c|c|c|c|c|}
\hline \multirow{2}{*}{ Type of laboratory test } & \multirow{2}{*}{ Result } & \multicolumn{2}{|c|}{ Sample type } & \multirow{2}{*}{$\begin{array}{c}\text { All samples } \\
\text { n (\%) }\end{array}$} \\
\hline & & $\begin{array}{l}\text { Respiratory }{ }^{\mathrm{a}} \\
\mathrm{n}(\%)\end{array}$ & $\begin{array}{c}\text { Non-respiratory } \\
n(\%)\end{array}$ & \\
\hline \multirow{3}{*}{ Smear microscopy } & All & $7,320(100)$ & $816(100)$ & $8,176(100)$ \\
\hline & Positive & $181(2.5)$ & $82(10.0)$ & $268(3.3)$ \\
\hline & Negative & $7,139(97.5)$ & $734(90.0)$ & $7,908(96.7)$ \\
\hline \multirow{6}{*}{ Culture $^{c}$} & All & $7,749(100)$ & $1,015(100)$ & $8,806(100)$ \\
\hline & Positive $^{d}$ & $392(5.1)$ & $142(14.0)$ & $541(6.1)$ \\
\hline & Positive: $M T B(C)^{e}$ & 149 (1.9) & $40(3.9)$ & $195(2.2)$ \\
\hline & Positive: NTM & $211(2.7)$ & $89(8.8)$ & $314(3.6)$ \\
\hline & Negative & $7,213(93.1)$ & $848(83.5)$ & 8,096 (91.9) \\
\hline & Contaminated & $144(1.8)$ & $25(2.5)$ & 169 (1.9) \\
\hline \multirow{4}{*}{$\begin{array}{l}\text { Direct rapid molecular } \\
\text { identification of } M T B(C) \text { in } \\
\text { primary samples }^{f}\end{array}$} & All & $308(100)$ & $258(100)$ & $578(100)$ \\
\hline & Positive for MTB(C)e & $64(20.8)$ & $28(10.8)$ & $93(16.1)$ \\
\hline & Negative & $242(78.6)$ & $218(84.5)$ & $471(81.5)$ \\
\hline & Indeterminate & $2(0.6)$ & $12(4 \cdot 7)$ & $14(2.4)$ \\
\hline \multirow{4}{*}{$\begin{array}{l}\text { Direct rapid molecular DST } \\
\text { on primary samples }\end{array}$} & All & $64(100)$ & $28(100)$ & $93(100)$ \\
\hline & Rifampicin susceptible & $59(92.2)$ & $26(92.9)$ & $85(91.4)$ \\
\hline & Rifampicin resistant & $4(6.2)$ & $2(7.1)$ & $7(7 \cdot 5)$ \\
\hline & Indeterminate & $1(1.6)$ & $0(0.0)$ & $1(1.1)$ \\
\hline
\end{tabular}

BCG: Bacillus Calmette-Guérin; DST: drug susceptibility testing; MTB(C): Mycobacterium tuberculosis complex; NTM: non-tuberculous mycobacteria.

a Respiratory samples: sputum, gastric aspirate, bronchoalveloar lavage, pleural fluid, other respiratory samples (e.g. nasopharyngeal aspirate); non-respiratory samples: cerebrospinal fluid, lymph node, pus, other biopsy tissue, urine, other non-respiratory samples (e.g. pericardial fluid).

b Including samples of unknown type.

Either Löwenstein-Jensen, Mycobacteria Growth Indicator Tube or other solid or liquid media. Only one positive culture result per sample was analysed.

d 32 of the 541 positive culture samples were either not further identified or were identified as M. bovis BCG.

e $M$. bovis BCG not included.

Used the following assays: Croatia: GeneXpert MTB/RIF, Amplified MTB Direct Test GenProbe; Germany: GeneXpert MTB/RIF, GenoType MTBDRplus, in-house test; Italy: GeneXpert MTB/RIF, INNO-LiPA; Latvia: GeneXpert MTB/RIF; Lithuania: GeneXpert MTB/RIF; United Kingdom: GeneXpert MTB/RIF, GenoType MTBDRplus, INNO-LiPA, IS6110 sequencing.

Almost all $(8,806 / 9,157,96.2 \%)$ samples were cultured and a positive culture was obtained in $541(6.1 \%)$ of the 8,806 primary samples. Culture was more often positive from non-respiratory than from respiratory samples $(142 / 1,015,14.0 \%$ vs 392/7,749, 5.1\%, p<0.0001 for all positive cultures; $40 / 1,015,3.9 \%$ vs $149 / 7,749$, $1.9 \%, p=0.0001$ for MTB(C)-positive cultures) (Table 2 ).

Across all sites, the culture contamination rate (on solid and liquid media) was 169/8,806 (1.9\%) (Table 2), ranging from $0 / 2,884$ (o\%) at the Croatian to $16 / 240$ $(6.7 \%)$ at the German site.

Per-sample analysis showed that MTB(C) was detected in $236 / 8,851(2.7 \%)$ of primary samples with the highest rates at the German and UK sites, 23/275 (8.4\%) and $107 / 2,132(5.0 \%)$, respectively (Table 3 ). The sample positivity rates for MTB(C) were significantly higher in pus ( $p=0.0005), \operatorname{LN}(p<0.0001), \operatorname{CSF}(p=0.002)$ and other tissue biopsy $(p=0.002)$ samples compared with sputum samples. Among respiratory samples, MTB(C) was more commonly detected in GA samples than in sputum samples $(43 / 1,231,3.5 \%$ vs $89 / 4,684$, $1.9 \%, p=0.002)$. No statistically significant difference between $\mathrm{MTB}(\mathrm{C})$ detection rates in GA or BAL samples was observed. Per-patient analysis showed that MTB(C) was isolated in 156/5,156 (3.0\%) of children (Table 4). MTB(C) was detected in $31 / 643(4.8 \%)$ patients who submitted GA samples; of them, 21/31 patients submitted at least two GA samples. The first GA sample identified 16 positive patients, the second identified an additional four and the third, another positive patient.

Multidrug resistance (MDR), defined as resistance to at least rifampicin and isoniazid, was seen in 10/156 $(6.4 \%)$ of patients ranging from $0 / 23$ and $0 / 4$ at Croatian 


\section{TABLE 3}

Laboratory diagnosis of paediatric tuberculosis: paediatric primary samples identified as MTB(C) across five study sites, $2007-2011(n=236)$

\begin{tabular}{|c|c|c|c|c|c|c|c|c|c|}
\hline \multirow{2}{*}{ Site } & \multicolumn{9}{|c|}{ Number of samples identified as $\mathrm{MTB}(\mathrm{C})^{\mathrm{a}} /$ total number per sample type (\%) } \\
\hline & Sputum & GA & $\mathrm{BAL}$ & Lymph node & Pus & CSF & $\begin{array}{l}\text { Tissue } \\
\text { biopsy }\end{array}$ & $\begin{array}{c}\text { Other } \\
\text { samples }\end{array}$ & All samples \\
\hline $\begin{array}{l}\text { Croatia } \\
\text { CIPH }\end{array}$ & $6 / 1,402(0.4)$ & $20 / 694(2.9)$ & $9 / 516(1.7)$ & $1 / 20(5.0)$ & $0 / 0(0.0)$ & $0 / 19(0.0)$ & $0 / 3(0.0)$ & $1 / 138(0.7)$ & $37 / 2,792(1.3)$ \\
\hline $\begin{array}{l}\text { Germany } \\
\text { NRCM }\end{array}$ & $1 / 29(3.4)$ & $9 / 53(17.0)$ & $5 / 39(12.8)$ & $2 / 58(3.4)$ & $0 / 1(0.0)$ & $1 / 13(7.7)$ & $2 / 35(5 \cdot 7)$ & $3 / 47(6.4)$ & 46/2,397 (1.9) \\
\hline $\begin{array}{l}\text { Latvia } \\
\text { NTRL }\end{array}$ & $16 / 1,219(1.3)$ & $4 / 378(1.1)$ & $13 / 546(2.4)$ & $1 / 2(50.0)$ & $1 / 10(10.0)$ & 1/9 (11.1) & $6 / 135(4.4)$ & 4/98 (4.1) & $107 / 2,132(5.0)$ \\
\hline $\begin{array}{l}\text { Lithuania } \\
\text { TBL }\end{array}$ & $13 / 1,099(1.2)$ & o/17 (0.0) & $2 / 54(3.7)$ & $4 / 40(10.0)$ & $0 / 1(0.0)$ & $0 / 6(0.0)$ & $1 / 1(100)$ & $3 / 37(8.1)$ & $23 / 1,255(1.8)$ \\
\hline $\begin{array}{l}\text { United Kingdom } \\
\text { NMRL }\end{array}$ & $53 / 935(5.7)$ & $10 / 89(11.2)$ & $7 / 312(2.2)$ & $6 / 83(7.2)$ & $8 / 102(7.8)$ & $7 / 91(7.7)$ & 6/56 (10.7) & $10 / 464(2.2)$ & $107 / 2,132(5.0)$ \\
\hline All sites & $89 / 4,684(1.9)$ & $43 / 1,231(3.5)$ & $36 / 1,467(2.5)$ & $14 / 203(6.9)$ & $9 / 114(7.9)$ & $9 / 138(6.5)$ & $15 / 230(6.5)$ & $21 / 784(2.7)$ & $236 / 8,851(2.7)$ \\
\hline
\end{tabular}

BAL: bronchoalveolar lavage; BCG: Bacillus Calmette-Guérin; CIPH: Croatian National Institute of Public Health; National Mycobacterium Reference Laboratory; CSF: cerebrospinal fluid; GA: gastric aspirate; MTB(C): Mycobacterium tuberculosis complex; NMRL: National Mycobacterium Reference Laboratory; NRCM: National Reference Centre for Mycobacteria at Forschungszentrum Borstel; NTRL: National TB Reference Laboratory at the Latvian Infectology Centre; TBL: Tuberculosis Bacteriology Laboratory at the Infectious Diseases and Tuberculosis Hospital.

a By isolation of $\operatorname{MTB}(C)$ culture or identification of $M T B(C)$ nucleic acid in a sample. M. bovis BCG results are not included.

b Data from 2011 only.

\section{TABLE 4}

Laboratory-confirmed paediatric cases ${ }^{\mathrm{a}}$ across the six study sites (with primary samples only), 2007-2011 (n=156)

\begin{tabular}{|c|c|c|c|c|}
\hline \multirow{3}{*}{ Site } & \multicolumn{2}{|c|}{ Type of primary sample } & \multirow{3}{*}{$\begin{array}{c}\text { Identification of MTB(C) } \\
\mathrm{n} \text { MTB(C)-positive } \\
\text { patients } / \mathrm{N} \text { patients } \\
(\%)\end{array}$} & \multirow{3}{*}{$\begin{array}{c}\text { Drug susceptibility } \\
\text { testing } \\
\text { n with MDR/ N MTB(C)- } \\
\text { positive patients }{ }^{\mathrm{c}} \\
(\%)\end{array}$} \\
\hline & Sputum & Gastric aspirate & & \\
\hline & $\begin{array}{l}\text { n samples/ N patients } \\
\text { (ratio) }\end{array}$ & $\begin{array}{l}\text { n samples/ N patients } \\
\text { (ratio) }\end{array}$ & & \\
\hline $\begin{array}{l}\text { Croatia } \\
\text { CIPH }\end{array}$ & $1,402 / 1,006(1.4)$ & $694 / 391(1.8)$ & $23 / 1,681(1.4)$ & $0 / 23(0.0)$ \\
\hline $\begin{array}{l}\text { Germany } \\
\text { NRCM }\end{array}$ & $29 / 21(1.4)$ & $53 / 22(2.4)$ & $7 / 152(4.6)$ & $1 / 7(14 \cdot 3)$ \\
\hline $\begin{array}{l}\text { Italy }^{\mathrm{b}} \\
\text { HSR }\end{array}$ & NA & NA & $4 / 200(2.0)$ & $0 / 4(0.0)$ \\
\hline $\begin{array}{l}\text { Latvia } \\
\text { NTRL }\end{array}$ & $1,219 / 504(2.4)$ & $378 / 170(2.2)$ & $40 / 1,151(3.5)$ & $3 / 40(7.5)$ \\
\hline $\begin{array}{l}\text { Lithuania } \\
\text { TBL }\end{array}$ & $1,385 / 774(1.8)$ & $18 / 13(1.4)$ & $17 / 886$ (1.9) & $3 / 17(17.7)$ \\
\hline $\begin{array}{l}\text { United Kingdom } \\
\text { NMRL }\end{array}$ & $939 / 382(2.5)$ & $89 / 47$ (1.9) & $65 / 1,086(6.0)$ & $3 / 65(4.6)$ \\
\hline All sites & $4,974 / 2,687$ (1.9) & $1,232 / 643$ (1.9) & $156 / 5,156(3.0)$ & $10 / 156(6.4)$ \\
\hline
\end{tabular}

CIPH: Croatian National Institute of Public Health, National Mycobacterium Reference Laboratory; HSR: San Raffaele Scientific Institute in collaboration with the Institute 'Villa Marelli', NiguardaCa' Granda Hospital;MDR: multidrug resistance (resistant to rifampicin and isoniazid); NA: data not available; NMRL: National Mycobacterium Reference Laboratory; MTB(C): Mycobacterium tuberculosis complex; NRCM: National Reference Centre for Mycobacteria at Forschungszentrum Borstel; NTRL: National TB Reference Laboratory at the Latvian Infectology Centre; TBL: Tuberculosis Bacteriology Laboratory at the Infectious Diseases and Tuberculosis Hospital.

a By isolation of $\operatorname{MTB}(C)$ culture or identification of $M T B(C)$ nucleic acid from any of the patient's samples.

b Data from 2011 only.

c $M$. bovis BCG not included. 
Laboratory diagnosis of paediatric tuberculosis: performance of molecular assays compared with culture in detecting MTB(C) in primary samples across the six study sites ${ }^{\text {a }}$,2007-2011

\begin{tabular}{|c|c|c|c|c|c|c|c|c|c|c|}
\hline \multirow{2}{*}{$\begin{array}{l}\text { Sample type } \\
\text { and smear results }\end{array}$} & \multicolumn{2}{|c|}{ Sensitivity } & \multicolumn{2}{|c|}{ Specificity $^{c}$} & \multicolumn{2}{|c|}{$\begin{array}{l}\text { Positive predictive } \\
\text { value }^{d}\end{array}$} & \multicolumn{2}{|c|}{$\begin{array}{l}\text { Negative predictive } \\
\text { value }^{\mathrm{e}}\end{array}$} & \multicolumn{2}{|c|}{ Accuracy ${ }^{f}$} \\
\hline & $\mathrm{n} / \mathrm{N}$ & $\%(95 \% \mathrm{Cl})$ & $\mathrm{n} / \mathrm{N}$ & $\%(95 \% \mathrm{Cl})$ & $\mathrm{n} / \mathrm{N}$ & $\%(95 \% \mathrm{Cl})$ & $\mathrm{n} / \mathrm{N}$ & $\%(95 \% \mathrm{Cl})$ & $\mathrm{n} / \mathrm{N}$ & $\%(95 \% \mathrm{Cl})$ \\
\hline All ${ }^{g, h}$ & $57 / 72$ & $\begin{array}{c}79.2 \\
(68.0-87.8) \\
\end{array}$ & $411 / 439$ & $\begin{array}{c}93.6 \\
(90.9-95.7) \\
\end{array}$ & $57 / 85$ & $\begin{array}{c}67.1 \\
(56.0-76.9) \\
\end{array}$ & $411 / 426$ & $\begin{array}{c}96.5 \\
(94.3-98.0) \\
\end{array}$ & $468 / 511$ & $\begin{array}{c}91.6 \\
(88.8-93.8) \\
\end{array}$ \\
\hline $\begin{array}{l}\text { All smear } \\
\text { positives }\end{array}$ & $42 / 46$ & $\begin{array}{c}91.3 \\
(79.2-97.6) \\
\end{array}$ & $41 / 56$ & $\begin{array}{c}73.2 \\
(59.7-84.2) \\
\end{array}$ & $42 / 67$ & $\begin{array}{c}62.7 \\
(60.3-84.5) \\
\end{array}$ & $41 / 45$ & $\begin{array}{c}91.1 \\
(78.8-97.5) \\
\end{array}$ & $83 / 102$ & $\begin{array}{c}81.4 \\
(72.4-88.4) \\
\end{array}$ \\
\hline $\begin{array}{l}\text { All smear } \\
\text { negatives }\end{array}$ & $11 / 21$ & $\begin{array}{c}52.4 \\
(29.8-74.3) \\
\end{array}$ & $287 / 299$ & $\begin{array}{c}96.0 \\
(93.1-97.9) \\
\end{array}$ & $11 / 23$ & $\begin{array}{c}47.8 \\
(26.8-69.4)\end{array}$ & $287 / 297$ & $\begin{array}{c}96.6 \\
(93.9-98.4)\end{array}$ & $298 / 320$ & $\begin{array}{c}93.1 \\
(89.8-95.6)\end{array}$ \\
\hline Respiratory ${ }^{g}$ & $48 / 54$ & $\begin{array}{c}88.9 \\
(77.4-95.8)\end{array}$ & $224 / 236$ & $\begin{array}{c}94.9 \\
(91.3-97.3)\end{array}$ & $48 / 60$ & $\begin{array}{c}80.0 \\
(67.7-89.2)\end{array}$ & $224 / 230$ & $\begin{array}{c}97.4 \\
(94.4-99.0)\end{array}$ & $272 / 290$ & $\begin{array}{c}93.8 \\
(90.4-96.3)\end{array}$ \\
\hline $\begin{array}{l}\text { Respiratory } \\
\text { smear positives }\end{array}$ & $40 / 42$ & $\begin{array}{c}95.2 \\
(83.8-99.4)\end{array}$ & $15 / 20$ & $\begin{array}{c}75.0 \\
(50.9-91.3) \\
\end{array}$ & $40 / 45$ & $\begin{array}{c}88.9 \\
(75.9-96.3) \\
\end{array}$ & $15 / 17$ & $\begin{array}{c}88.2 \\
(63.6-98.5) \\
\end{array}$ & $55 / 62$ & $\begin{array}{c}88.7 \\
(78.1-95.4) \\
\end{array}$ \\
\hline $\begin{array}{l}\text { Respiratory } \\
\text { smear negatives }\end{array}$ & $5 / 8$ & $\begin{array}{c}62.5 \\
(24.5-91.5) \\
\end{array}$ & $161 / 168$ & $\begin{array}{c}95.8 \\
(91.6-98.3) \\
\end{array}$ & $5 / 12$ & $\begin{array}{c}41.7 \\
(15.2-72.3) \\
\end{array}$ & $161 / 164$ & $\begin{array}{c}98.2 \\
(94.7-99.6)\end{array}$ & $166 / 176$ & $\begin{array}{c}94.3 \\
(89.8-97.2) \\
\end{array}$ \\
\hline Non-respiratory & $7 / 16$ & $\begin{array}{c}43.8 \\
(19.8-70.1)\end{array}$ & $177 / 192$ & $\begin{array}{c}92.2 \\
(87.4-95.6) \\
\end{array}$ & $7 / 22$ & $\begin{array}{c}31.8 \\
(13.9-54.9)\end{array}$ & $177 / 186$ & $\begin{array}{c}95.2 \\
(91.0-97.8)\end{array}$ & $184 / 208$ & $\begin{array}{c}88.5 \\
(83.3-92.5) \\
\end{array}$ \\
\hline $\begin{array}{l}\text { Non-respiratory } \\
\text { smear positives }\end{array}$ & $2 / 4$ & $\begin{array}{c}50.0 \\
(6.8-93.2) \\
\end{array}$ & $24 / 34$ & $\begin{array}{c}70.6 \\
(52.5-84.9) \\
\end{array}$ & $2 / 12$ & $\begin{array}{c}16.7 \\
(2.1-48.4) \\
\end{array}$ & $24 / 26$ & $\begin{array}{c}92.3 \\
(74.9-99.1) \\
\end{array}$ & $26 / 38$ & $\begin{array}{c}68.4 \\
(51.3-82.5) \\
\end{array}$ \\
\hline $\begin{array}{l}\text { Non-respiratory } \\
\text { smear negatives }\end{array}$ & $5 / 12$ & $\begin{array}{c}41.7 \\
(15.2-72.3) \\
\end{array}$ & $118 / 123$ & $\begin{array}{c}95.9 \\
(90.8-98.7)\end{array}$ & $5 / 10$ & $\begin{array}{c}50.0 \\
(18.7-81.3) \\
\end{array}$ & $118 / 125$ & $\begin{array}{c}94.4 \\
(88.8-97.7)\end{array}$ & $123 / 135$ & $\begin{array}{c}91.1 \\
(85 \cdot 0-95 \cdot 3)\end{array}$ \\
\hline Sputum ${ }^{g}$ & $34 / 37$ & $\begin{array}{c}91.9 \\
(78.1-98.3) \\
\end{array}$ & $54 / 57$ & $\begin{array}{c}94.7 \\
(85.4-98.9 \\
\end{array}$ & $34 / 37$ & $\begin{array}{c}91.9 \\
(78.1-98.3) \\
\end{array}$ & $54 / 57$ & $\begin{array}{c}94.7 \\
(85.4-98.9 \\
\end{array}$ & $88 / 94$ & $\begin{array}{c}93.6 \\
(86.6-97.6 \\
\end{array}$ \\
\hline $\begin{array}{l}\text { Sputum } \\
\text { smear positives }\end{array}$ & $32 / 33$ & $\begin{array}{c}97.0 \\
(84.2-99.9) \\
\end{array}$ & $6 / 9$ & $\begin{array}{c}66.7 \\
(29.9-92.5) \\
\end{array}$ & $32 / 35$ & $\begin{array}{c}91.4 \\
(76.9-98.2) \\
\end{array}$ & $6 / 7$ & $\begin{array}{c}85.7 \\
(42.1-99.6 \\
\end{array}$ & $38 / 42$ & $\begin{array}{c}90.5 \\
(77.3-97.3 \\
\end{array}$ \\
\hline $\begin{array}{l}\text { Sputum } \\
\text { smear negatives }\end{array}$ & $1 / 3$ & $\begin{array}{c}33.3 \\
(0.8-90.6) \\
\end{array}$ & $48 / 48$ & $\begin{array}{c}100 \\
(92.6-100) \\
\end{array}$ & $1 / 1$ & $\begin{array}{c}100 \\
(2.5-100) \\
\end{array}$ & $48 / 50$ & $\begin{array}{c}96.0 \\
(86.3-99.5) \\
\end{array}$ & $49 / 51$ & $\begin{array}{c}96.0 \\
(86.5-99.5) \\
\end{array}$ \\
\hline Gastric aspirates ${ }^{g}$ & $8 / 10$ & $\begin{array}{c}80.0 \\
(44.4-97.5)\end{array}$ & 95/99 & $\begin{array}{c}96.0 \\
(90.0-98.9)\end{array}$ & $8 / 12$ & $\begin{array}{c}66.7 \\
(34.9-90.1)\end{array}$ & $95 / 97$ & $\begin{array}{c}97.9 \\
(92.7-99.7)\end{array}$ & $103 / 109$ & $\begin{array}{c}94.5 \\
(88.4-97.9)\end{array}$ \\
\hline $\begin{array}{l}\text { Bronchoalveolar } \\
\text { lavage }^{\mathrm{g}}\end{array}$ & $6 / 7$ & $\begin{array}{c}85.7 \\
(42.1-99.6) \\
\end{array}$ & $55 / 59$ & $\begin{array}{c}93.2 \\
(83.5-98.1) \\
\end{array}$ & $6 / 10$ & $\begin{array}{c}60.0 \\
(26.2-87.8) \\
\end{array}$ & $55 / 56$ & $\begin{array}{c}98.2 \\
(90.4-100) \\
\end{array}$ & $61 / 66$ & $\begin{array}{c}92.4 \\
(83.2-97.5) \\
\end{array}$ \\
\hline
\end{tabular}

$\mathrm{Cl}$ : confidence interval; MTB(C): Mycobacterium tuberculosis complex.

a CIPH: Croatian National Institute of Public Health, National Mycobacterium Reference Laboratory; HSR: San Raffaele Scientific Institute in collaboration with the Institute 'Villa Marelli', NiguardaCa' Granda Hospital; NMRL: National Mycobacterium Reference Laboratory; NRCM: National Reference Centre for Mycobacteria at Forschungszentrum Borstel; NTRL: National TB Reference Laboratory at the Latvian Infectology Centre; TBL: Tuberculosis Bacteriology Laboratory at the Infectious Diseases and Tuberculosis Hospital.

b Number of true positives/number of true positives + number of false negatives.

c Number of true negatives/number of true negatives + number of false positives.

Number of true positives/number of true positives + number of false positives.

Number of true negatives/number of true negatives + number of false negatives.

f Number of true positives + number of true negatives/total number of tested samples.

g Number of smear negatives + number of smear positives + number with smear result unknown.

h Number of respiratory samples + number of non-respiratory samples + number of samples of unknown type.

and Italian sites to $3 / 17$ of cases at the Lithuanian site (Table 4).

Rapid molecular test performance for Mycobacterium tuberculosis complex and drug-resistance detection Routine use of NAAT was initiated in 1996 at the Croatian, in 1994 at the German and Italian, in 2010 at the Latvian, in 2008 at the Lithuanian and in 1999 at the UK sites. A variety of commercial and in-house assays were used on $578 / 9,157(6.3 \%)$ of samples.
The bacteria in respiratory samples were more often directly identified as $\mathrm{MTB}(\mathrm{C})$ than those in non-respiratory samples $(64 / 308,20.8 \%$ vs $28 / 258,10.9 \%$, $\mathrm{p}=0.001)$. The rate of indeterminate results was higher among non-respiratory samples $(12 / 258,4.7 \%$ vs 2/308, 0.6\%, $p=0.0024$ ) (Table 2 ).

A total of 511 primary samples were tested by both molecular assay and culture for MTB(C) identification. Compared with liquid or solid culture, a rapid molecular test - based on the pooled data analysis - had an overall (for all samples types) sensitivity of 
$57 / 72(79.2 \%)$, specificity of $411 / 439$ (93.6\%), PPV of $57 / 85(67.1 \%)$, NPV of $411 / 426(96.5 \%)$ and accuracy of $468 / 511$ (91.6\%) for MTB(C) detection (Table 5). The sensitivity of molecular tests for smear-positive samples vs smear-negative samples was significantly higher $(42 / 46,91.3 \%$ vs. $11 / 21,52.4 \%, p=0.0006)$, while they were less specific $(41 / 56,73.2 \%$ vs $287 / 299$, $96.0 \%, p=0.0001)$. The lower specificity in the smearpositive samples was due to 12 false-positive results at the UK site tested by INNO-LiPA Rif.TB (Innogenetics, Ghent, Belgium) and Xpert MTB/RIF, two at the German site tested by Xpert MTB/RIF and one at Croatian site tested by Xpert MTB/RIF.

The molecular tests detected MTB(C) in 48/54 (88.9\%) of respiratory and $7 / 16$ (43.8\%) of non-respiratory samples $(p=0.0004)$; the specificity was $224 / 236(94.9 \%)$ and $177 / 192(92.2 \%)$, respectively. For both respiratory and non-respiratory samples, the sensitivity was again higher for smear-positive than for smear-negative samples; however, the specificity was higher for smearnegative samples for both groups of samples (Table 5).

Molecular assays produced $15 / 511$ (2.9\%) of falsenegative results and $28 / 511(5.5 \%)$ of false-positive results for $M T B(C)$ identification; discordant results were related to all types of samples. Mutations coding resistance to rifampicin were detected in $7 / 93$ (7.5\%) of all primary samples tested by rapid assays (Table 2 ). Molecular and phenotypic assays were fully concordant with DST.

Non-tuberculous mycobacteria and Mycobacterium bovis $\mathrm{BCG}$

Approximately half $(989 / 1,903,52.0 \%)$ of all positive isolates (grown from primary samples and those received as reference cultures) were identified as NTMs at the Croatian (43/94), German (54/90), Italian (26/50) and UK $(862 / 1,564,55.1 \%)$ sites. NTMs were less common at the Latvian (1/67) and Lithuanian (3/38) sites. A proportion of all positive isolates (224/1,903, 11.8\%) were identified as $M$. bovis BCG.

NTMs were isolated at the highest rate from LN samples (27/203, 13.3\%). NTMs were seen in $136 / 4,958$ (2.7\%) of all paediatric patients, with M. abscessus as the most frequently identified.

\section{Discussion}

This large study reviewed the analysis of over 10,000 samples tested across a number of European TB reference laboratories and showed the availability and use of all conventional and modern diagnostic techniques across these settings. Although the diagnostic algorithms were similar across laboratories, approaches towards collecting paediatric non-respiratory samples differ between European sites, with very few samples other than sputum obtained from children in Latvia and Lithuania.
Because young children can rarely expectorate but instead swallow their sputum, aspiration of the gastric content is the best (and the least unpleasant) procedure for obtaining a sputum sample suitable for further diagnostics $[4,5,19,24]$. Our study showed that, compared with sputum samples, testing GA more often results in positive findings. Recent data by Fiebig et al. support this evidence [25]: they report widespread use of GA in Germany and a high (63\%) yield of culture and NAAT in paediatric specimens, confirming that it is possible to reliably diagnose TB in children and disagreeing with the common notion of 'difficulties' in laboratory confirmation of paediatric disease.

Our data suggest that collection of several GA samples might help identify further cases, in line with previously published data [5]. Larger studies are needed to assess the differences in diagnostic yield in serial samples.

Extrapulmonary samples also tested positive more frequently than sputum samples did, placing emphasis on the usefulness of collecting non-respiratory materials, in line with some previously published results that showed a good performance of fine-needle aspiration biopsy of LN in confirming TB [26]. Considering the observed small proportion of non-respiratory samples received at the Latvian and Lithuanian sites, it would seem reasonable to expect a low incidence of extrapulmonary TB there. However, crude comparison with World Health Organization (WHO) surveillance data for 2011 showed that while in Croatia, Germany, Italy and the UK extrapulmonary TB represented the minority of paediatric cases $(15 \%, 17 \%, 19 \%$ and $37 \%$, respectively), the majority of notified cases in Latvia and Lithuania were, in contrast, extrapulmonary (59\% and $92 \%$, respectively) [23], pointing towards predominantly non-laboratory based diagnosing. Within the framework of our study, infrequent collection of samples other than sputum might be explained by differences in the referral population, with a large number of asymptomatic children being screened for TB as part of contact tracing in medium-incidence settings, such as those of the participating Baltic countries [23]. Relatively low MTB(C)-positivity rates at the Latvian and Lithuanian sites also reflect this screening strategy. There, a lower level of clinical suspicion might be the reason for not carrying out more invasive procedures. Another explanation might be a more cautious attitude of eastern European physicians in Latvia and Lithuania in collecting GA or non-respiratory samples from children, related to the higher invasiveness of these procedures compared with sputum collection, and lack of confidence in the success of laboratory diagnosis. The rates of notified extrapulmonary TB across the EU/EEA vary between $4 \%$ and $48 \%(37.7 \%$ in children during 2002 to 2011), possibly reflecting the challenges and differences in diagnosis and confirming the urgent need for improvement $[27,28]$. 
Analysis of the performance of the pooled molecular tests showed that the sensitivity was comparable to that reported for the Xpert MTB/RIF in children in other studies: $79.5 \%$ for sputum and GA in Vietnam, 90.0 and $68.8 \%$ for sputum and GA samples in Zambia, $73.6 \%$ and $75.9 \%$ in South Africa using NPA and sputum samples, $54.7 \%$ in Tanzania [4,6,17-19]. The molecular tests were more sensitive in detecting $\mathrm{MTB}(\mathrm{C})$ in respiratory samples than in non-respiratory samples as previously described $[17,29]$ which may be explained by the design of the molecular tests originally aimed to work in respiratory samples. We observed no differences in sensitivity and specificity for sputum, GA, or BAL samples, as previously observed [19].

The specificity was lower in smear-positive samples than in smear-negative samples. It might be an artefact resulting from the pooling of different molecular methods or the low specificity of molecular tests for the DNA target or the use of an imperfect gold standard method. Although culture is the recognised current gold standard for TB diagnosis, it still might be an imperfect comparator, possibly leading to failure to diagnose some cases [17].

Similar performance characteristics of INNO-LiPA Rif. TB and Xpert MTB/RIF used for the diagnosis of TB in adults were reported in Italy and the UK [7,29]; however, these results were primarily evaluating a single rapid method (Xpert MTB/RIF) on a single type of sample. High incidence of TB may explain the higher PPV observed in studies conducted outside Europe $(100 \%$ for sputum and GA in Vietnam, $81.8 \%$ and $86.8 \%$ for sputum and GA samples respectively in Zambia, $92.8 \%$ and $89.7 \%$ in South Africa using NPA and sputum samples) $[6,17-19]$, compared with our study.

Our study showed that NTMs were more frequently isolated from children than MTB(C). M. bovis BCG was frequently isolated in Latvia and Lithuania where BCG vaccination is a national policy. Our data showed that $M$. abscessus was the predominant NTM isolated from children while previously was the M. avium complex [30].

This study has several limitations. The analysis was based on subnational data, leading to possible selection bias and restriction of the geographical representativeness of the study. A diagnostic bias resulting from more invasive samples being taken when there is a higher level of clinical suspicion may cause the higher positivity rates in non-respiratory samples. Two sites could provide 2011 data only. Small case numbers forced 'pooling' of the NAAT results and our approach ignores the possible heterogeneity of results or differences in ROC curve of various tests. The retrospective laboratory-based approach did not allow the investigators to obtain data on HIV status, clinical/radiological findings and TB treatment. A more comprehensive assessment of the success of diagnosing TB in children in Europe is needed. Ideally, new studies should be led by key organisations in the field of infectious diseases control and prevention, such as ECDC or WHO Regional Office for Europe, and result in effective recommendations.

As long as available evidence for paediatric TB diagnosis remains limited, however, this study is of public health importance as it reviewed routine laboratory work in a non-clinical trial context, allowing conclusions to be drawn based on real-life scenarios. Moreover, the study sites represent the largest laboratories with reference functions. This work has also demonstrated that data from the ERLN-TB can be used to conduct operational research.

Despite the relative success in diagnosing TB when using non-respiratory or GA samples, the level of laboratory confirmation in children remains low, resulting in treatment initiation based on a set of subjective parameters. TB treatment is long and antituberculosis drugs have potential toxicity; therefore while it is important not to risk failing to diagnose TB, overdiagnosis may result in unnecessary psychological or physical stress for children [26]. With this in mind, a more intensive approach to obtaining paediatric samples, including samples other than sputum (in particular GA and non-respiratory samples), is advisable in order to increase the number of laboratory-confirmed cases and give physicians a much greater degree of confidence when administering antituberculosis treatment. Additionally, a major effort is needed to optimise and evaluate molecular assays for analysis of GA or nonrespiratory samples, thus making the diagnosis of paediatric TB more accurate.

European Reference Laboratory Network (ERLN-TB) members

Vanessa Mathys, National Reference Centre of Tuberculosis and Mycobacteria, Communicable \& Infectious Diseases, Scientific Institute of Public Health, Brussels, Belgium; Elizabeta Bachiyska, National Center of Infectious and Parasitic Diseases, Sofia, Bulgaria; Despo PieridouBagatzouni, National Reference Laboratory for Mycobacteria, Nicosia General Hospital, Cyprus; Ilona Zemanová, Mycobacteriology Unit and National Reference Laboratory for Mycobacteria NIPH, Prague, Czech Republic; Tiina Kummik, TB Reference Laboratory, Department of Mycobacteriology, Tartu University Hospital, Tartu, Estonia; Merja Marjamäki, National Institute for Health and Welfare, THL, Turku, Finland; Sabine Rüsch-Gerdes, National Reference Center for Mycobacteria, Forschungszentrum Borstel, Borstel, Germany; Dimitrios Papaventsis, National Reference Laboratory for Mycobacteria (NRLM), Sotiria Chest Diseases Hospital, Athens, Greece; Panayotis Ioannidis, National Reference Laboratory for Mycobacteria (NRLM), Sotiria Chest Diseases Hospital, Athens, Greece; Nóra Szabó, National TB Reference Laboratory, Budapest, Hungary; Diego Zallocco, S. Raffaele Scientific Institute, Milan, Italy; Luigi Codecasa, Regional Reference Centre for Tuberculosis, Institute "Villa Marelli”, Niguarda Ca' Granda Hospital, Milan, Italy; Ester Mazzola, Regional Reference Laboratory for Tuberculosis, Niguarda Ca' Granda Hospital, Milan, Italy; Turid Mannsåker, National Reference Laboratory for Mycobacteria, Norwegian Institute of Public Health, Division of Infectious Disease 
Control, Oslo, Norway; Anabela Santos-Silva, National Tuberculosis Reference Laboratory, National Institute of Health (INSA), Porto, Portugal; Manca Žolnir-Dovč, National Reference Laboratory for Mycobacteria, University Clinic for Respiratory and Allergic Diseases, Golnik, Slovenia; Sofia Samper, Hospital Universitario Miguel Servet, IIS Aragón, Zaragoza, Spain; Maria Soledad-Jimenez, Mycobacterial Reference Laboratory, Servicio de Bacteriología, Centro Nacional de Microbiología, Instituto de Salud Carlos III, Madrid, Spain; Kristian Ängeby, Clinical Microbiology, MTC, Karolinska Institute, Karolinska University Hospital, Stockholm, Sweden.

\section{Conflict of interest}

None declared.

\section{Acknowledgements}

We are grateful to Brian Woodward, Vladyslav Nikolayevskyy, Noha Seoudi, Doris Hillemann and Mihaela Obrovac for the precious help in data collection. We thank Csaba Ködmön for providing surveillance data helpful for building study conclusions. We are grateful to Basel Karo, Kristin Tolksdorf, Göran Kirchner and Manuel Dehnert for the help in data cleaning and in statistical analysis. We thank Barbara Hauer and Yvan Hutin for their input in the study, and Aftab Jasir and Steen Ethelberg for critical reading of the manuscript.

\section{Authors' contributions}

Conceived and designed the study: AS, LF, FD, WH, CDM, YB. Collected data: AS, LF, WH, FD, ER, VKJ, EP, GS, CDM, ERLNTB members, YB. Analysed the data: AS, LF, WH, FD, ER, VKJ, EP, GS, CDM, YB. Wrote the paper: AS, YB. All authors read and approved the final manuscript.

\section{References}

1. World Health Organization (WHO). Global tuberculosis report 2012. Geneva: WHO; 2012, Available from: http://www.who. int/tb/publications/global_report/gtbr12_main.pdf

2. Sandgren A, Cuevas LE, Dara M, Gie RP, Grzemska M, Hawkridge A, et al. Childhood tuberculosis: progress requires an advocacy strategy now. Eur Respir J. 2012;40(2):294-7. http://dx.doi.org/10.1183/09031936.00187711

3. Basu Roy R, Whittaker E, Kampmann B. Current understanding of the immune response to tuberculosis in children. Curr Opin Infect Dis. 2012;25(3):250-7. http://dx.doi.org/10.1097/ QCO.ob013e3283529af9

4. Zar HJ, Connell TG, Nicol M. Diagnosis of pulmonary tuberculosis in children: new advances. Expert Rev Anti Infect Ther. 2010;8(3):277-88. http://dx.doi.org/10.1586/eri.10.9

5. Cruz TA, Revell PA, Starke JR. Gastric aspirate yield for children with suspected pulmonary tuberculosis. J Ped Infect Dis. 2013;2(2):171-4

6. Zar HJ, Workman L, Isaacs W, Munro J, Black F, Eley B, et al. Rapid molecular diagnosis of pulmonary tuberculosis in children using nasopharyngeal specimens. Clin Infect Dis. 2012;55(8):1088-95. http://dx.doi.org/10.1093/cid/cis598

7. Tortoli E, Russo C, Piersimoni C, Mazzola E, Dal Monte $P$, Pascarella $M$, et al. Clinical validation of Xpert MTB RIF for the diagnosis of extrapulmonary tuberculosis. Eur Respir J. 2012;40(2):442-7. http://dx.doi. org/10.1183/09031936.00176311

8. Drobniewski F, Nikolayevskyy V, Balabanova Y, Bang D, Papaventsis D. Diagnosis of tuberculosis and drug resistance: what can new tools bring us? Int J Tuberc Lung Dis. 2012;16(7):860-70. http://dx.doi.org/10.5588/ijtld.12.0180

9. Drobniewski F, Nikolayevskyy V, Maxeiner H, Balabanova Y, Casali N, Kontsevaya I, et al. Rapid diagnostics of tuberculosis and drug resistance in the industrialized world: clinical and public health benefits and barriers to implementation. BMC Med. 2013;11:190. http://dx.doi.org/10.1186/1741-7015-11-19o

10. World Health Organization (WHO). Multidrug and extensively drug-resistant TB (M/XDR-TB): 2010 global report on surveillance and response. Geneva: WHO; 2010. Available from http://whqlibdoc.who.int/publications/2010/9789241599191_ eng.pdf

11. Balabanova Y, Coker R, Fedorin I, Zakharova S, Plavinskij $\mathrm{S}$, Krukov N, et al. Variability in interpretation of chest radiographs among Russian clinicians and implications for screening programmes: observational study. BMJ. 2005;331(7513):379-82. http://dx.doi.org/10.1136/ bmj.331.7513.379

12. Cuevas LE, Browning R, Bossuyt $P$, Casenghi M, Cotton MF, Cruz AT, et al. Evaluation of tuberculosis diagnostics in children: 2. Methodological issues for conducting and reporting research evaluations of tuberculosis diagnostics for intrathoracic tuberculosis in children. Consensus from an expert panel. J Infect Dis. 2012;205 Suppl2:S209-15. http:// dx.doi.org/10.1093/infdis/jir879

13. World Health Organization (WHO). Policy statement: automated real-time nucleic acid amplification technology for rapid and simultaneous detection of tuberculosis and rifampicin resistance: Xpert MTB/RIF system. Geneva: WHO; 2011. Available from: http://whqlibdoc.who.int/ publications/2011/9789241501545_eng.pdf

14. Boehme CC, Nabeta P, Hillemann D, Nicol MP, Shenai S, Krapp $\mathrm{F}$, et al. Rapid molecular detection of tuberculosis and rifampin resistance. N Engl J Med. 2010;363(11):1005-15. http://dx.doi. org/10.1056/NEJMoao907847

15. Hillemann D, Rüsch-Gerdes S, Boehme C, Richter E. Rapid molecular detection of extrapulmonary tuberculosis by the automated GeneXpert MTB/RIF system. J Clin Microbiol. 2011;49(4):1202-5. http://dx.doi.org/10.1128/JCM.02268-10

16. Steingart KR, Sohn H, Schiller I, Kloda LA, Boehme CC, Pai M, et al. Xpert $®$ MTB/RIF assay for pulmonary tuberculosis and rifampicin resistance in adults. Cochrane Database Syst Rev. 2013;1:CDo09593.

17. Nicol MP, Workman L, Isaacs W, Munro J, Black F, Eley B, et al. Accuracy of the Xpert MTB/RIF test for the diagnosis of pulmonary tuberculosis in children admitted to hospita in Cape Town, South Africa: a descriptive study. Lancet Infect Dis. 2011;11(11):819-24. http://dx.doi.org/10.1016/ S1473-3099(11)70167-0

18. Nhu NT, Ha DT, Anh ND, Thu DD, Duong TN, Quang ND, et al. Evaluation of Xpert MTB/RIF and MODS assay for the diagnosis of paediatric tuberculosis. BMC Infect Dis. 2013;13:31. http:// dx.doi.org/10.1186/1471-2334-13-31

19. Bates M, O'Grady J, Maeurer M, Tembo J, Chilukutu L, Chabala $C$, et al. Assessment of the Xpert MTB/RIF assay for diagnosis of tuberculosis with gastric lavage aspirates in children in sub-Saharan Africa: a prospective descriptive study. Lancet Infect Dis. 2013;13(1):36-42. http://dx.doi.org/10.1016/ S1473-3099(12)70245-1

20. Sandgren A, Hollo V, Quinten C, Manissero D. Childhood tuberculosis in the European Union/European Economic Area, 2000 to 2009. Euro Surveill. 2011;16(12): pii=19825.

21. ERLN-TB. The European Reference Laboratory Network for TB. [Accessed 12 Jan 2012]. Available from: http://www.erlntb.eu/

22. Drobniewski FA, Nikolayevskyy V, Hoffner S, Pogoryelova 0 , Manissero D, Ozin AJ. The added value of a European Union tuberculosis reference laboratory network--analysis of the national reference laboratory activities. Euro Surveill. 2008;13(12): $\mathrm{pii}=8076$.

23. World Health Organization (WHO). Tuberculosis country profiles. Geneva: WHO. [Accessed 20 Sep 2012]. Available from: http://www.who.int/tb/country/data/profiles/en/

24. Zar HJ, Hanslo D, Apolles P, Swingler G, Hussey G. Induced sputum versus gastric lavage for microbiological confirmation of pulmonary tuberculosis in infants and young children: a prospective study. Lancet. 2005;365(9454):130-4. http:// dx.doi.org/10.1016/S0140-6736(05)17702-2

25. Fiebig L, Hauer B, Brodhun B, Balabanova Y, Haas W. Bacteriologic confirmation of pulmonary tuberculosis in children with gastric aspirates in Germany, 2002-2010. Int J Tuberc Lung Dis. Forthcoming 2014.

26. Perez-Velez CM, Marais BJ. Tuberculosis in children. N Engl J Med. 2012;367(4):348-61. http://dx.doi.org/10.1056/ NEJMra1008049

27. Solovic I, Jonsson J, Korzeniewska-Koseła M, Chiotan DI, Pace-Asciak A, Slump E, et al. Challenges in diagnosing extrapulmonary tuberculosis in the European Union, 2011. Euro Surveill. 2013;18(12): $\mathrm{pii}=20432$.

28. Sandgren A, Hollo V, van der Werf MJ. Extrapulmonary tuberculosis in the European Union and European Economic Area, 2002 to 2011. Euro Surveill. 2013;18(12): pii=20431.

29. Seoudi N, Mitchell SL, Brown TJ, Dashti F, Amin AK, Drobniewski FA. Rapid molecular detection of tuberculosis and rifampicin drug resistance: retrospective analysis of a national U.K. molecular service over the last decade. Thorax. 2012;67(4):361-7. http://dx.doi.org/10.1136/ thoraxjnl-2011-200610

30. Cook JL. Nontuberculous mycobacteria: opportunistic environmental pathogens for predisposed hosts. Br Med Bull. 2010;96:45-59. http://dx.doi.org/10.1093/bmb/ldq035 